\title{
Erratum to: A sequential GM-based PHD filter for a linear Gaussian system
}

\author{
LIU ZongXiang*, XIE WeiXin, WANG Pin \& YU You \\ ATR Key Laboratory, Shenzhen University, Shenzhen 518060, China
}

Erratum to: Science China Information Sciences, October 2013, Vol.56 102302:1-102302:10 doi: $10.1007 / \mathrm{s} 11432-013-4901-y$

The online version of this research paper unfortunately contained two mistakes. Author names were missing partially or completely from the authorship list and citation, respectively. These mistakes were accidentally caused by the incompatibility of the typesetting software. The printed version is correct as the above. 